Supporting Information for

\title{
Identification and Quantification of Any Isoforms of Carbohydrates by 2D UV-MS Fingerprinting of Cold Ions.
}

Erik Saparbaev, ${ }^{1}$ Vladimir Kopysov, ${ }^{1}$ Viktoriia Aladinskaia, ${ }^{1}$ Vincent Ferrieres, ${ }^{2}$ Laurent Legentil, $^{2}$ and Oleg V. Boyarkin ${ }^{1}$.

${ }^{1}$ Laboratoire de Chimie Physique Moléculaire, École Polytechnique Fédérale de Lausanne, Station-6, 1015 Lausanne, Switzerland

${ }^{2}$ Université de Rennes, Ecole Nationale Supérieure de Chimie de Rennes, CNRS, ISCR UMR 6226, F-35000 Rennes, France

*Correspondence to: oleg.boiarkin@epfl.ch

\section{Table of Contents}

Section S1. Details of apparatus and experimental procedure S1

Figure S1. UVPD spectra for complexes of $\mathrm{TyrH}^{+}$with tetrasaccharides ........................... S2

Figure S2 UVPD spectra for complexes of $\mathrm{TyrH}^{+}$with two heptasaccharides ................... S3

Figure S3 UVPD spectra for complexes of $\mathrm{TrmH}^{+}$with five disaccharides ........................ S4

Table S1. Tested solution mixtures of disaccharides ........................................................ S5 


\section{Details of apparatus and experimental procedure.}

The ions, produced in the gas phase from solution by a nano-electrospray ionization (n-ESI) source, enter an electrodynamic ion funnel (IF) orthogonally to its axis through a $100 \mathrm{~mm}$ long stainless steel capillary of $0.7 \mathrm{~mm}$ ID. The ions are then turned by $90^{\circ}$ to travel along the IF axis at pressure of 6 mbar and exit the funnel through the conductance limit of $2.5 \mathrm{~mm}$ diameter. They subsequently pass through two skimmers (1.5 and $2 \mathrm{~mm}$ in diameter), which allow for a gradual reduction of pressure from 0.5 mbar to $10^{-4}$ mbar. The ions are then accumulated and thermalized in an octupole ion trap for $97 \mathrm{~ms}$, before they are released and pass through a quadrupole mass filter (Q1), which is set to select the ions of interest. Mass-selected ions are then turned by $90^{\circ}$ using an electrostatic bender, focused by a stack of three electrostatic lenses and moved through an RF octupole guide into a cold octupole trap, ${ }^{33}$ which is kept at $6 \mathrm{~K}$. The trap is driven by two $1 \mathrm{MHz}$ sinus waveforms with peak-to-peak amplitudes of 50-100 V. The stored in the trap non-covalent complexes are cooled down to $\sim 10 \mathrm{~K}$ in collisions with He buffer gas, ${ }^{33}$ which is pulsed into the trap shortly before arrival of the ions. Approximately $85 \mathrm{~ms}$ later the ions are irradiated by a pulse of UV light $(1.8 \pm 0.7$ $\mathrm{mJ} /$ pulse, $5 \mathrm{~ns}$ duration, $\sim 6 \mathrm{~cm}^{-1}$ spectral linewidth), produced by a UV optical parametric oscillator (OPO; NT 342C, EKSPLA) and undergo photo fragmentation. The fragment and parent ions were $90^{\circ}$ turned by the second electrostatic quadrupole bender either toward a highly sensitive quadrupole mass spectrometer (QMS) or toward high-resolution broadband Orbitrap-based MS (Exactive, Thermo Fisher). UVPD spectra and 2D UV-MS fingerprints are measured by continuously recording the yield of the photofragments using the QMS and the Orbitrap-based MS, respectively, while scanning UV wavelength. The repetition rate of the cooling/fragmentation cycle was determined by the $10 \mathrm{~Hz}$ repetition rate of the OPO, such that the parent ions experienced only one OPO shot in each cycle. At each UV wavelength the yield for a single fragment (with QMS) or the entire fragment mass spectrum was measured in 10 cycles and averaged to give a data point in UVPD spectrum or a fragment MS in 2D UVMS fingerprint, respectively.

Special measures have been taken to narrow the variation of pulse energy of the OPO over the wide tuning range (210-350 $\mathrm{nm})$. The angular positions of non-linear crystals in the OPO doubling/mixing stages were detuned from their optimal phasematching angles, such that the output energy at each wavelength was close to the 
minimum energy attainable within the desired spectral range. This minimizes the potential contributions to UVPD from non-linear absorption and therefore increases reproducibility of the fingerprints. In addition, the measured ion signals were normalized to OPO pulse energy, which was measured by a broadband pyroelectric detector.

In measurements with Orbitrap-MS, the whole fragment mass spectrum was normalized to the total ion signal detected in the cycle. In QMS measurements we employ $20 \mathrm{~Hz}$ cooling cycle and detect fragment or parent ions in $10 \mathrm{~Hz}$ alternative cycles with and without UV pulse, respectively. 
a)

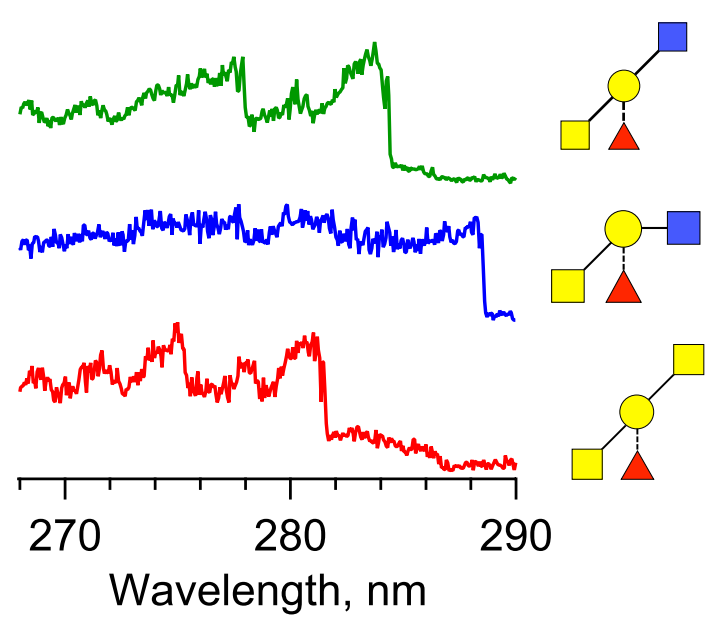

b)
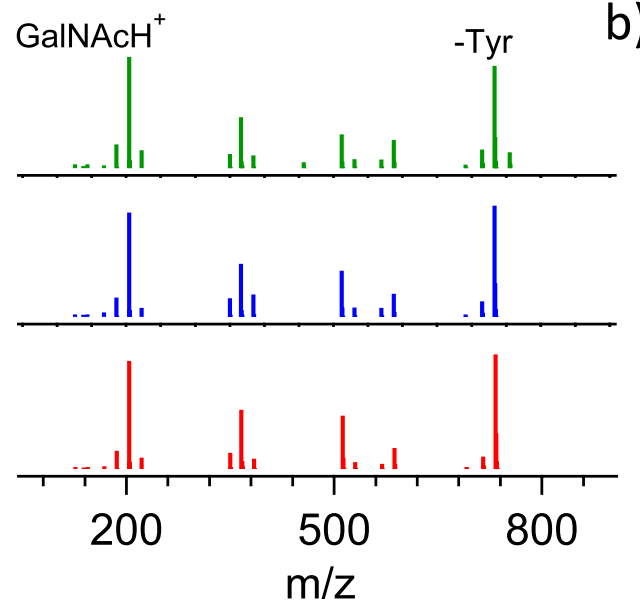

Figure S1. UVPD (a) optical and (b) mass spectra of $\mathrm{TyrH}^{+}$in complexes (from top to bottom) with Blood Type A1, Blood Type A2 and Blood Type A13/4 tetrasaccharides. The spectra are obtained by integrating the respective 2D UV-MS fingerprints over $\mathrm{m} / \mathrm{z}$ and wavelength dimensions for optical and mass spectra, respectively. 
a)

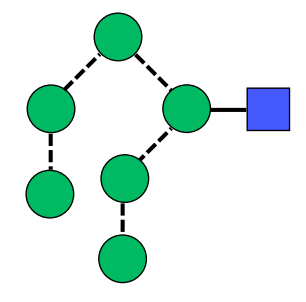

c)

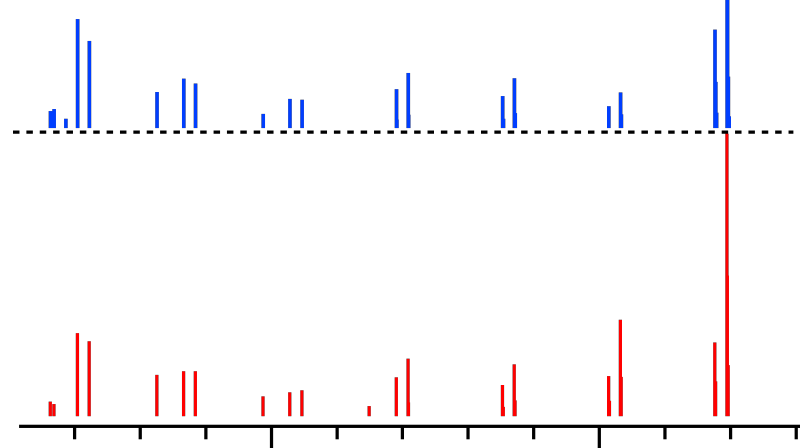

500

d)

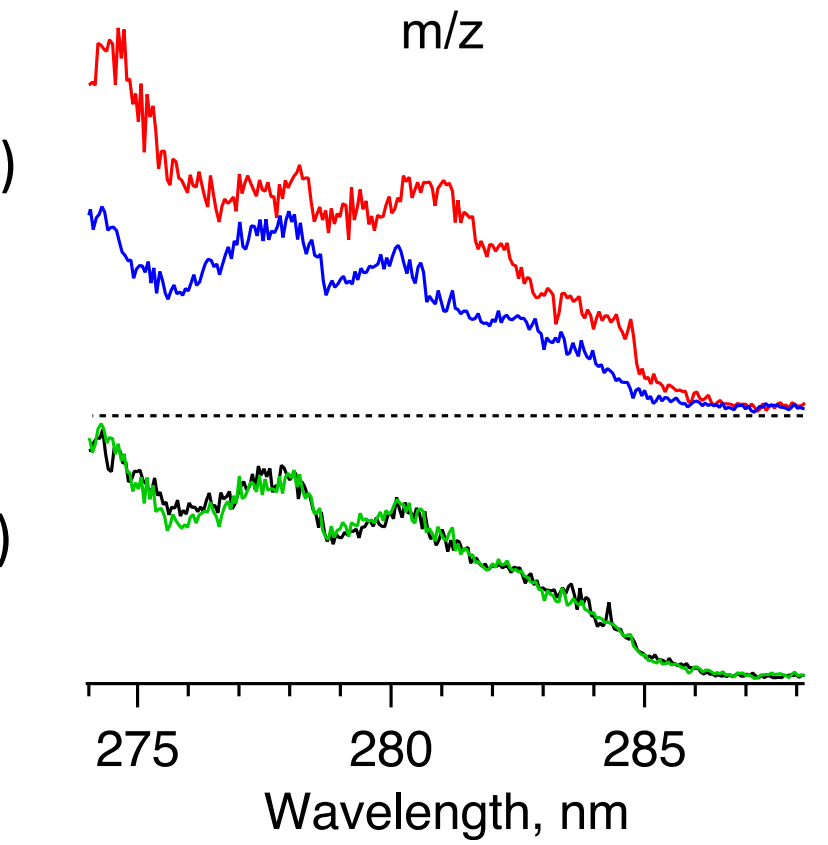

Figure S2. Symbolic structures of (a) Man 6 GlcNAc-II and (b) Man 6 GlcNAc-I hepta saccharides. (c) UVPD mass spectra (in red and blue, respectively) and (d) UVPD optical spectra (the same colour coding) of the two heptasaccharides in complexes with $\mathrm{TyrH}^{+}$; the spectra are derived by integrating the respective measured 2D UV-MS fingerprints. (e) UVPD optical spectrum (green trace) derived from the 2D UV-MS fingerprint measured for 1:2 mixture of the glycans and the best fit of these spectrum (red trace) obtained from matrix decomposition procedure. The calculated relative concentrations, $29 \%$ and $71 \%$, differ from the prepared solution ones by $4 \%$. 


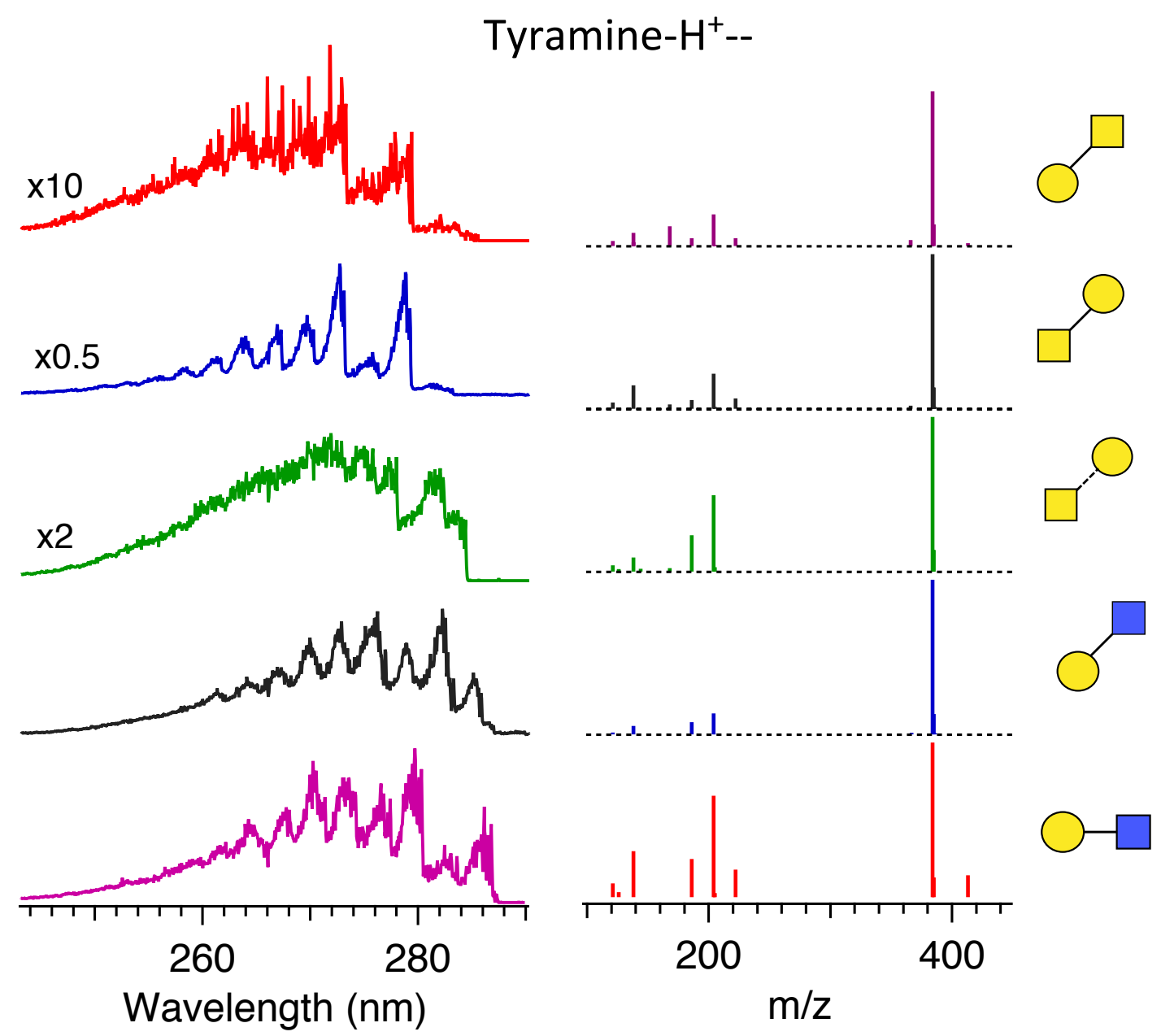

Figure S3. UVPD optical and MS identities of five isomeric disaccharides in non-covalent complexes with $\mathrm{TrmH}^{+}$aromatic molecule. 
Calculated relative concentrations

\begin{tabular}{|c|c|c|c|c|c|}
\hline Sixture & Aaccharide & B & C & D & E \\
\hline B2:C1 & 0.00 & 78.30 & 17.15 & 4.55 & 0.00 \\
\hline B1:C1 & 0.00 & 58.85 & 36.65 & 4.50 & 0.00 \\
\hline B1:C2 & 0.00 & 34.40 & 60.25 & 5.35 & 0.00 \\
\hline A2:C1 & 63.17 & 1.93 & 29.80 & 5.10 & 0.00 \\
\hline A1:C1 & 50.60 & 1.30 & 42.10 & 5.99 & 0.00 \\
\hline A1:C2 & 48.24 & 0.05 & 49.30 & 2.41 & 0.00 \\
\hline D1:E1 & 0.00 & 1.22 & 0.00 & 51.26 & 47.52 \\
\hline B1:D1:E1 & 0.00 & 33.57 & 0.12 & 34.38 & 31.93 \\
\hline B1:D2:E1 & 0.00 & 25.60 & 0.00 & 51.25 & 23.14 \\
\hline B1:D1:E2 & 0.00 & 22.18 & 0.00 & 30.30 & 47.52 \\
\hline E & 0.00 & 1.85 & 0.00 & 0.00 & 98.15 \\
\hline D & 0.00 & 2.29 & 6.19 & 91.52 & 0.00 \\
\hline
\end{tabular}

Table S1. Calculated relative concentrations for 12 mixtures of one to three isomeric disaccharides from the 5-component library of individual disaccharides.

The identities of the isomers are encoded by A-E letters, where A - $\beta$-Gal-1,3-GalNAc, B - $\alpha$ GalNAc-1,3-Gal, C - $\beta$-GalNAc-1,3-Gal, D - $\beta$-Gal-1,3-GlcNAc, E - $\beta$-Gal-1,4-GlcNAc; the relative solution concentrations of the mixed isomers are shown next to the letters. The leftmost column lists the tested mixtures of disaccharides and the other five columns show the relative concentrations of the five isomers calculated from matrix decompositions. 